EPJ Web of Conferences 102, 00005 (2015)

DOI: $10.1051 /$ epjconf/ 201510200005

(C) Owned by the authors, published by EDP Sciences, 2015

\title{
Dust Opacities
}

\author{
Michiel Min \\ ${ }^{1}$ Anton Pannekoek Institute, University of Amsterdam, The Netherlands
}

\begin{abstract}
Dust particles are the dominant source of opacity at (almost) all wavelengths and in (almost) all regions of protoplanetary disks. By this they govern the transport of energy through the disk and thus the thermal structure. Furthermore, their spectral properties determine the low resolution spectral signature observed at infrared wavelengths. The infrared resonances that can be observed using low resolution infrared spectroscopy can be used to identify the composition and size distribution of the dust. The opacities depend on the size, shape, structure and composition of the particles, and computing them is not always trivial. In this chapter the ways in which the opacity of dust particles depend on the dust characteristics is discussed. Methods to compute them are outlined and the difficulties that can be encountered are addressed.
\end{abstract}

\section{Introduction}

Dust grains in cosmic environments obscure, scatter, and reprocess radiation. This means that a proper understanding of environments containing dust requires detailed knowledge of these processes. In addition, studying radiation that interacted with dust grains can teach us about the properties of the particles. In protoplanetary disks, dust grains are the main source of opacity at most wavelengths. They cause these disks to be heavily optically thick, and determine the radiation field and temperature structure in most parts of the disk. Thereby, they are also important for the dynamics of the disk.

The way in which dust particles interact with light depends on the dust particle characteristics like size, shape, and composition. Computations of the optical properties of dust particles thus require us to take these properties correctly into account. Unfortunately, this is not always feasible. Small scale substructure interior or at the surface of small grains require very high spatial resolution in the simulations that is usually not computationally feasible. Especially for large particles this becomes a significant issue, since it is required to both model the large scale structure of the particle, and the small scale substructure, since they can act on different aspects of the optical properties.

Laboratory measurements of the properties of cosmic dust grains are essential for understanding their optical properties for two important reasons. The first is that in all computations the first thing we have to know is the refractive index of the material. The wavelength dependent refractive index is very different for different material compositions and lattice structures. Therefore, measurements of the refractive index of a large set of materials relevant for cosmic dust is the essential first requirement for modeling any dusty structure. The second reason why laboratory measurements on cosmic dust

$\star 4^{\text {th }}$ Lecture from Summer School "Protoplanetary Disks: Theory and Modelling Meet Observations"

This is an Open Access article distributed under the terms of the Creative Commons Attribution License 4.0, which permits unrestricted use, distribution, and reproduction in any medium, provided the original work is properly cited. 
are important is that our computational methods are limited, and it is essential that they are gauged by comparing to measured optical properties of realistic particles.

Over the past our understanding of how small particles interact with light has increased significantly. This topic is driven from many different fields of research. Many of the available computational tools where developed for atmospheric research, biological research, or astronomy. However, also in industrial applications there is a large interest in this, for example in the paint industry, or the field of characterization of nano particles.

In this chapter we will focus on creating an understanding of the complexity of the problem, and what issues one has to think of. The purpose is to create awareness of the difficulties associated with the optical properties of dust particles and provide solutions to handle these issues properly when modeling or interpreting observations. Many of the insights and ideas discussed in this chapter come from the excellent books on this topic by Bohren \& Huffman (1983); van de Hulst (1957) and from Draine (1988); Min (2009); Voshchinnikov et al. (2007) and other papers by these authors.

\section{Interaction of light with a particle: the basics}

The interaction of a light beam with a particle is usually divided into absorption and scattering. Absorbed radiation can be transformed into thermal energy or used for chemical alteration or evaporation of the particle. Scattered radiation retains its original wavelength, but changes direction.

\subsection{Interaction cross sections}

We have to consider the effective cross sections for scattering and absorption of radiation. These quantities depend on the characteristics of the particle and on the wavelength of radiation. There are several quantities related to the cross sections of absorption, scattering, and extinction, $C_{\mathrm{abs}}, C_{\mathrm{sca}}$, $C_{\text {ext }}=C_{\text {abs }}+C_{\text {sca }}$, that can be found in the literature. First there is the commonly used efficiency,

$$
Q_{\mathrm{abs}, \mathrm{sca}, \mathrm{ext}}=\frac{C_{\mathrm{abs}, \mathrm{sca}, \mathrm{ext}}}{2 \pi r},
$$

where $r$ is the size of the particle. This size is easily defined when the particle is a homogeneous sphere, but it becomes less transparent when the particle is irregularly shaped. In this case the size measure that is commonly used is the volume equivalent radius, $r_{V}$,

$$
r_{V}=\left(\frac{3 V}{4 \pi}\right)^{1 / 3},
$$

where $\mathrm{V}$ is the material volume of the particle, i.e. it is the radius of a sphere with the same material volume as the particle. There are two length scales involved in the scattering problem; the wavelength, $\lambda$, and the size of the particle, $r_{V}$. Therefore, it is convenient to use the size parameter,

$$
x=k r_{V}
$$

where $k=2 \pi / \lambda$. Due to scaling invariance of Maxwell's equations, solutions with the same size parameter are equivalent.

Another commonly used quantity is the mass absorption, scattering, or extinction cross section,

$$
\kappa_{\text {abs,sca,ext }}=\frac{C_{\text {abs,sca,ext }}}{M},
$$

where $M$ is the total mass of the particle. This is the quantity we will mainly use in practice, since it has some nice properties as we will see later on.

Because of Kirchhoff's law for emission and absorption, the emission cross section of a particle is equal to its absorption cross section. 


\subsection{Scattering of light and Stokes vectors}

Consider a monochromatic lightbeam traveling in a plane. We can divide the electric field into a component parallel to the plane, $E_{\|}$, and a component perpendicular to the plane, $E_{\perp}$. This light beam can be fully represented by the Stokes vector,

$$
\mathbf{S}=\left(\begin{array}{c}
I \\
Q \\
U \\
V
\end{array}\right)=\left(\begin{array}{c}
E_{\|} E_{\|}^{*}+E_{\perp} E_{\perp}^{*} \\
E_{\|} E_{\|}^{*}-E_{\perp} E_{\perp}^{*} \\
E_{\|} E_{\perp}^{*}+E_{\perp} E_{\|}^{*} \\
i\left(E_{\|} E_{\perp}^{*}-E_{\perp} E_{\|}^{*}\right)
\end{array}\right)
$$

The intensity of the light wave is represented by the Stokes parameter $I$. The degree of linear polarization is $\sqrt{Q^{2}+U^{2}} / I$, and the degree of circular polarization is $V / I$. The strength of the Stokes vector becomes apparent when one wants to write down what happens when a light beam interacts with a particle. Upon interaction, the state of the light beam is changed. When we place a detector somewhere in the plane defined above, the scattered radiation detected due to interaction with the particle can be obtained through the scattering matrix, $\mathbf{F}$,

$$
\left(\begin{array}{c}
I \\
Q \\
U \\
V
\end{array}\right)_{\text {scat }}=\left(\begin{array}{llll}
F_{11} & F_{12} & F_{13} & F_{14} \\
F_{21} & F_{22} & F_{23} & F_{24} \\
F_{31} & F_{32} & F_{33} & F_{34} \\
F_{41} & F_{42} & F_{43} & F_{44}
\end{array}\right)\left(\begin{array}{c}
I \\
Q \\
U \\
V
\end{array}\right)_{\text {inc }} .
$$

In principle the scattering matrix depends on the scattering angle $\theta$, which is the angle between the incident and scattered direction, and azimuthal direction, i.e. the angle between the inertial frame of the particle and the scattering plane. The scattering matrix can not only be defined for a single particle, but also for a collection of particles. The scattering matrix simplifies significantly when the collection of particles obeys two simple rules: 1) they are in random orientation, and 2) for every particle the mirror particle exists. When this is the case, the scattering matrix becomes (van de Hulst 1957),

$$
\mathbf{F}=\left(\begin{array}{cccc}
F_{11} & F_{12} & 0 & 0 \\
F_{21} & F_{22} & 0 & 0 \\
0 & 0 & F_{33} & F_{34} \\
0 & 0 & -F_{34} & F_{44}
\end{array}\right)
$$

so there are only 6 elements left. This assumption is often used in radiative transfer computations. Also, in this case the scattering matrix only depends on the scattering angle, i.e. the angle between incoming and scattered light, and no longer on the orientation of the scattering plane with respect to the inertial frame of the particle.

\section{Computational methods}

To compute the cross sections and scattering matrix of a (collection of) particles one has to solve Maxwell's equations. There are numerous numerical methods to do this for various different particle shapes and sizes. Each of these methods has their advantages and disadvantages. Here we will discuss the most commonly used methods for optical properties of dust in protoplanetary disks. 


\subsection{Mie theory}

The simplest particle shape one can think of is that of a homogeneous sphere. The solution for the interaction of an electromagnetic wave with a homogeneous, spherical particle was first derived by Gustav Mie (Mie 1908). It is a very basic separation of variables technique, separating the angular and radial parts of Maxwell's equations. For a derivation and the mathematical details please see e.g. Bohren \& Huffman (1983). Mie theory provides a very stable method to compute the optical properties numerically, and there are numerous codes available that do this in a robust way. The solution involves an infinite sum which converges typically after $N=|m| x$ terms, with $m$ the complex refractive index of the material and $x$ the size parameter. Thus, Mie theory becomes more computationally challenging for large particles, small wavelengths, or highly conducting materials.

The term Mie theory or Mie scattering, is widely used in the literature to also refer to the solution for a radially stratified sphere, since the solution method is basically the same. It is even sometimes, erroneously, used for interaction of any type of particle with size comparable to the wavelength of radiation.

\subsection{Rayleigh approximation, small particles}

When the particle is much smaller than the wavelength of radiation both inside and outside the particle, i.e. $x \ll 1$ and $x|m| \ll 1$, the problem simplifies significantly. In this case, we can assume that the incoming electric field is homogeneous over the extend of the particle, the so-called electrostatic approximation. A particle will respond to this homogeneous electric field like a single ideal dipole. The dipole moment induced by the applied electric field $\mathbf{E}$ is given by (for a derivation see Chapter 5 of Bohren \& Huffman 1983),

$$
\mathbf{p}=\alpha \mathbf{E}_{\text {inc }},
$$

where $\alpha$ is the polarizability, i.e. the ease with which the particle is polarized. The cross sections for absorption and scattering can be directly derived from the polarizability,

$$
\begin{aligned}
& C_{\text {abs }}=k \operatorname{Im}(\alpha), \\
& C_{\text {sca }}=\frac{k^{4}}{6 \pi}|\alpha|^{2} .
\end{aligned}
$$

There are exact solutions for the polarizability of only a few particle shapes; spheres, ellipsoids, and layered spheres. The polarizability of an ellipsoid with the electric field applied along one of the axis is given by,

$$
\alpha=V \frac{m^{2}-1}{1+L\left(m^{2}-1\right)},
$$

with $0<L<1$ the form factor of the ellipsoid, depending on the axes ratios of the three different axes. There are three different form factors $L_{1,2,3}$ for the field applied along the different axes of the ellipsoid. The form factor has the property that $L_{1}+L_{2}+L_{3}=1$. For a homogeneous sphere $L_{1}=L_{2}=L_{3}=1 / 3$ and the polarizability becomes,

$$
\alpha=3 V \frac{m^{2}-1}{m^{2}+2} .
$$

It now also becomes clear why it is convenient to use the mass absorption cross section, $\kappa_{\mathrm{abs}}$. In the limit of very small particles, the cross section, $C_{\mathrm{abs}}$, is proportional to the volume of the particle, and thus to the mass. Therefore, in this limit, the mass absorption cross section is independent of particle size. 


\subsection{Effective medium theory}

Up to now we have only considered the case where the particle is composed of a single material with refractive index $m$. In practice, however, this is often not the case. There are numerical methods that can solve for complex systems with multiple materials (like the DDA discussed below), but a first order method to treat mixtures of materials is through the effective medium theory. In effective medium theory it is assumed that the mixing of materials takes place on the smallest possible scales, such that at any resolution one only observes an effective refractive index, $m_{\text {eff }}$. In this way the computational methods for single material particles can again be applied.

Consider an infinite medium with refractive index $m_{m}$ which we will call the matrix material. The polarizability of a small spherical inhomogeneity with refractive index $m_{i}$ in that medium is given by,

$$
\alpha_{i}=3 V \frac{\left(m_{i} / m_{m}\right)^{2}-1}{\left(m_{i} / m_{m}\right)^{2}+2} .
$$

Now the two most commonly used effective medium theories state that the polarizability of the effective medium inside this matrix material is the average of the polarizabilities of the materials that make up the medium,

$$
\frac{\left(m_{\mathrm{eff}} / m_{m}\right)^{2}-1}{\left(m_{\mathrm{eff}} / m_{m}\right)^{2}+2}=\sum_{i} w_{i} \frac{\left(m_{i} / m_{m}\right)^{2}-1}{\left(m_{i} / m_{m}\right)^{2}+2},
$$

where $w_{i}$ is the volume fraction of material $i$. To solve for $m_{\mathrm{eff}}$, the quantity which we want to derive, we have to define what the matrix material, $m_{m}$, is. In the Garnett mixing rule one choses the dominant material in the mixture, so $m_{m}=m_{j}$. This makes it possible to write down a closed form solution of Eq. 14, and is a good approximation if one wants to look at the effects of small abundance polutions in a material. In the Bruggeman mixing rule one makes the logical choice that $m_{m}=m_{\text {eff }}$ (so the left hand side of Eq. 14 becomes zero). This makes physically more sense, but also makes it more difficult to solve for $m_{\text {eff }}$. In practice however, a simple iterative scheme usually converges relatively fast. Note that using effective medium theory one can also quite easily add porosity to the particles by including a material with $m_{i}=1$.

It is good to realize the assumptions that go into effective medium theory. The most important one is that the mixing takes place at the smallest possible scale. For aggregates composed of constituents of different materials, this condition is obviously violated, since the constituents have finite size. Also, the assumption is that the inhomogeneities are spherical. This can have effects on the shapes of spectral resonances (see e.g. Min et al. 2008, for a discussion and proposed solution for this problem).

\subsection{Discrete Dipole Approximation (DDA)}

When we consider the particle to be build up from a large collection of very small sub-units, each of which interacts with the local electric field as a single dipole, we have (Draine 1988)

$$
\mathbf{p}_{j}=\alpha_{j}\left(\mathbf{E}_{\text {inc }, j}-\sum_{k \neq j}^{N} \mathbf{A}_{j k} \mathbf{p}_{k}\right),
$$

where $\mathbf{p}_{j}$ is the local dipole moment at the position of sub-unit $j, \alpha_{j}$ is the polarizability of sub-unit $j, \mathbf{E}_{\text {inc, } j}$ is the incoming field at the location of sub-unit $j$, and $N$ is the total number of sub-units. The $3 \times 3$ matrix $\mathbf{A}_{j k}$ determines the electric field at the position of sub-unit $j$ due to the dipole field emitted by sub-unit $k$ (Draine 1988).

$$
\mathbf{A}_{j k} \mathbf{p}_{k}=\frac{\exp \left(i k r_{j k}\right)}{4 \pi r_{j k}^{3}}\left\{\left(k r_{i j}\right)^{2} \hat{r}_{j k} \times\left(\hat{r}_{j k} \times \mathbf{p}_{k}\right)+\left(1-i k r_{j k}\right)\left(\mathbf{I}_{3}-3 \hat{\mathbf{r}}_{j k} \hat{\mathbf{r}}_{j k}\right) \mathbf{p}_{k}\right\}, \quad k \neq j,
$$


where $\hat{\mathbf{r}}_{i j}$ is the unit vector pointing from dipole $i$ to dipole $j, r_{i j}$ is the distance between these two dipoles, $\mathbf{I}_{3}$ is the $3 \times 3$ identity matrix, and the $3 \times 3$ matrix $\hat{\mathbf{r}}_{i j} \hat{\mathbf{r}}_{i j}$ is defined as the product of $\hat{\mathbf{r}}_{i j}$ as a column vector and $\hat{\mathbf{r}}_{i j}$ as a row vector, i.e.

$$
\hat{\mathbf{r}}_{i j} \hat{\mathbf{r}}_{i j}=\left[\begin{array}{ccc}
\hat{r}_{i j, x}^{2} & \hat{r}_{i j, x} \hat{r}_{i j, y} & \hat{r}_{i j, x} \hat{r}_{i j, z} \\
\hat{r}_{i j, x} \hat{r}_{i j, y} & \hat{r}_{i j, y}^{2} & \hat{r}_{i j, y} \hat{r}_{i j, z} \\
\hat{r}_{i j, x} \hat{r}_{i j, z} & \hat{r}_{i j, y} \hat{r}_{i j, z} & \hat{r}_{i j, z}^{2}
\end{array}\right],
$$

where $\hat{r}_{i j, x}, \hat{r}_{i j, y}$ and $\hat{r}_{i j, z}$ are the $x, y$ and $z$ components of the unit vector $\hat{\mathbf{r}}_{i j}$, respectively. It is clear that Eqs. 15 and 16 can be conveniently written as a single matrix equation.

$$
\tilde{\mathbf{E}}_{\text {inc }}=(\tilde{\mathbf{A}}+\tilde{\mathbf{B}}) \tilde{\mathbf{p}}
$$

where the $\tilde{\mathbf{E}}_{\text {inc }}$ and $\tilde{\mathbf{p}}$ are $3 N$ vectors containing the vectors $\mathbf{E}_{\text {inc, }, j}$ and $\mathbf{p}_{j}$ for all values of $j, \tilde{\mathbf{A}}$ is a $3 N \times 3 N$ matrix containing the matrices $\mathbf{A}_{i j}$ for all $i, j$, and $\tilde{\mathbf{B}}$ is a diagonal matrix with the values of $\alpha_{j}^{-1}$ on the diagonal. When this is solved for $\tilde{\mathbf{p}}$ (and thus for $\mathbf{p}_{j}$ ) the absorption cross section is given by,

$$
C_{\mathrm{abs}}=\frac{4 \pi k}{\left|E_{\mathrm{inc}}\right|^{2}} \sum_{j=1}^{N}\left\{\operatorname{Im}\left[\mathbf{p}_{j} \cdot\left(\alpha_{j}^{-1}\right)^{*} \mathbf{p}_{j}^{*}\right]-\frac{2}{3} k^{3} \mathbf{p}_{j}^{*} \cdot \mathbf{p}_{j}\right\},
$$

where the asterisks denote the complex conjugates. In the same way the other optical properties can be derived from the $\mathbf{p}_{j}$ (Draine 1988).

\subsubsection{DDA in the Rayleigh approximation}

In the limit of very small particles, i.e. very long wavelengths, Eq. 16 simplifies significantly,

$$
\mathbf{A}_{i j}=\frac{\mathbf{I}_{3}-3 \hat{\mathbf{r}}_{i j} \hat{\mathbf{r}}_{i j}}{4 \pi r_{i j}^{3}}, \quad i \neq j,
$$

which makes $\tilde{\mathbf{A}}$ real and symmetric. When we also assume the particle is homogeneous, i.e. $\tilde{\mathbf{B}}=\alpha^{-1} \mathbf{I}_{3 N}$, the solution for $\mathbf{p}_{j}$ can be written directly in terms of the eigenvalues and eigenvectors of $\tilde{\mathbf{A}}$. The derivation of this is beyond the scope of this chapter, but is relatively straightforward and elegant and the interested reader is referred to Min et al. (2006b) for all details. The final result is that the cross section of any particle of any shape can be written as a sum over the so-called form-factor distribution,

$$
C_{\mathrm{abs}}=\sum_{j=1}^{3 N} w_{j}\left[k V \operatorname{Im}\left(\frac{m^{2}-1}{1+L_{j}\left(m^{2}-1\right)}\right)\right],
$$

Here the values of $w_{j}$ are obtained from the eigenvectors of $\tilde{\mathbf{A}}$, and the form-factors $L_{j}$ from its eigenvalues. Comparing Eqs. 11 and 21 the surprising result is that the absorption cross section of any homogeneous particle in the Rayleigh limit can be exactly represented by a collection of ellipsoidal particles. This implies that the inversion problem, i.e. deriving the shape of the particle from its absorption cross section, is an inherently degenerate problem. For example it is not possible to uniquely derive the shape of the particles from the spectral shape of emission features. As we will see below, this becomes slightly better when we include particle size as a parameter and in addition consider the scattering properties of the particles as well. 


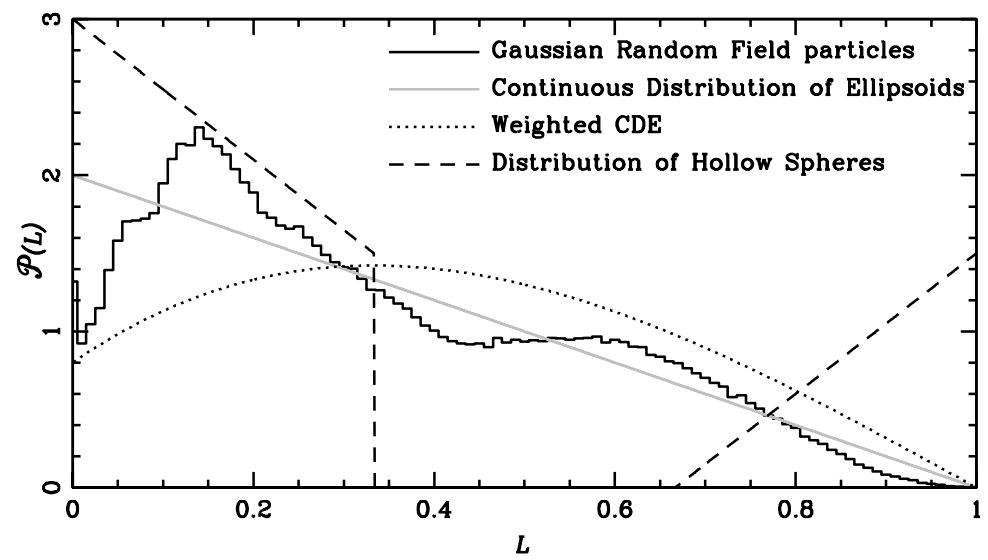

Figure 1. The form-factor distributions for different particle shape models used in the literature. Shown are the curves for irregularly shaped Gaussian Random Field particles (Min et al. 2007), the Continuous Distribution of Ellipsoids (CDE Bohren \& Huffman 1983), a weighted version of CDE (see e.g. Li 2008), and the Distribution of Hollow Spheres (DHS; Min et al. 2005).

In Fig. 1 a few form factor distributions are shown for different particle shapes. What is immediately clear is that there is almost always a strong contribution from very low value of $L$. These low values of $L$ correspond to extremely flattened or elongated ellipsoidal particles. This is not because the aspect ratio of the original particles is very high, but it represents sub-structure on the particle which has this behavior.

\subsection{The statistical approach}

The main idea of the statistical approach is to simplify the computation of optical properties but at the same time use a framework which is able to reduce the resonances introduced by oversimplifying the computations (for example by using homogeneous spherical particles). This idea was made popular by Bohren \& Huffman (1983) by introducing the so-called Continuous Distribution of Ellipsoids (CDE) for the analysis of infrared spectra (see also below).

As we have seen in the previous section, in the Rayleigh domain the absorption properties of any particle can be represented by those of a collection of ellipsoidal particles. This strongly supports the more general idea that the optical properties of a complex particle can be represented by those of a properly chosen collection of simple shapes. This has great computational advantages, since the optical properties of simple particle shapes can be computed much more efficiently. In addition, it allows a more strict focus on the particle characteristics that can be obtained from the observations.

Classically, spheroids have been a commonly used particle shape for applying the statistical approach (e.g. Dubovik et al. 2006; Fabian et al. 2001; Mishchenko et al. 1997, and many others). However, as discussed in the previous section, the aspect ratio that one has to use to reproduce the observed particle properties does not always represent the true particle shape. It can be that high aspect ratios are needed (low $L$ values) to reproduce rough surface substructure of an overall relatively spherical particle.

Another often applied approach is that of the distribution of hollow spheres (DHS; Min et al. 2003, 2005). This approach has the advantage that hollow spheres can be computed using Mie theory, and thus the computations are very easy and fast. As an interesting side-effect the rather exotic shape of 
a hollow sphere will not tempt anyone in interpreting the true particle shapes directly from the fitted distribution. Actually, the fact that DHS provides such a good fit to many observational properties of cosmic dust grains is an argument in itself that this interpretation of particle shapes should be done with great care.

In the DHS shape distribution we simply average over the fraction $f$ occupied by the central vacuum inclusion in the particle from zero to some value $f_{\max } \leq 1$, giving equal weights to all values of $f$ while the material volume of the particle is kept the same. This means that the particles with higher values of $f$ have a larger outer radius. Thus a particle with $f=0$ represents a homogeneous, solid sphere, while $f \approx 1$ represents a large, very thin bubble. The distribution is given by

$$
n(f)= \begin{cases}1 / f_{\max }, & 0 \leq f<f_{\max }, \\ 0, & f \geq f_{\max },\end{cases}
$$

where $n(f) d f$ is the fraction of the number of particles in the distribution between $f$ and $f+d f$.

The statistical approach has been often applied for spectroscopic analysis of solid state resonances. For this, usually particles much smaller than the wavelength dominate the spectra. Therefore, we can apply the Rayleigh limit. One of the most commonly used distributions of particle shapes is the Continuous Distribution of Ellipsoids. This is basically a form factor distribution with,

$$
w(L)=2-2 L, \quad 0<L<1 .
$$

The resulting absorption cross section is,

$$
C_{\mathrm{abs}}^{\mathrm{CDE}}=2 k V \operatorname{Im}\left(\frac{m^{2}}{m^{2}-1} \ln m^{2}\right) .
$$

CDE has been extremely successful in fitting infrared spectra of various mineralogical components in protoplanetary disks and dust around evolved stars and comets. Also for DHS with $f_{\max }=1$ there is a closed form solution for $C_{\text {abs }}$ in the Rayleigh limit (Min et al. 2003),

$$
C_{\mathrm{abs}}^{\mathrm{DHS}}=3 k V \operatorname{Im}\left(\frac{2 m^{2}+1}{2 m^{2}-2} \ln \left[\frac{\left(2 m^{2}+1\right)\left(m^{2}+2\right)}{9 m^{2}}\right]\right) .
$$

\section{Refractive index data}

There are generally speaking two different ways to obtain the refractive index of a material: 1) from reflection spectra of polished bulk material, and 2) from transmission spectra of a powder. The first method is direct and the most accurate. However, this is not always available for all materials. The second method requires a model for the particles to invert the powder spectrum into a refractive index spectrum, and this is far from unique. Therefore, it is crucial to check how the refractive index data one uses is obtained before applying different particle shape models to it.

Materials produced under slightly different conditions might display different optical properties. This is especially true for the so-called amorphous materials. The difference between an amorphous and a crystalline material is that in a crystalline material the atoms are ordered according to a well defined lattice structure. This lattice structure is the energetically most favorable organization of the atoms. In an amorphous material the formation temperature of the material was too low to allow the atoms to structure themselves into this energetically favorable lattice structure. Therefore, the lattice structure is un-organized to a certain level. Also, amorphous silicates more easily absorb impurities 


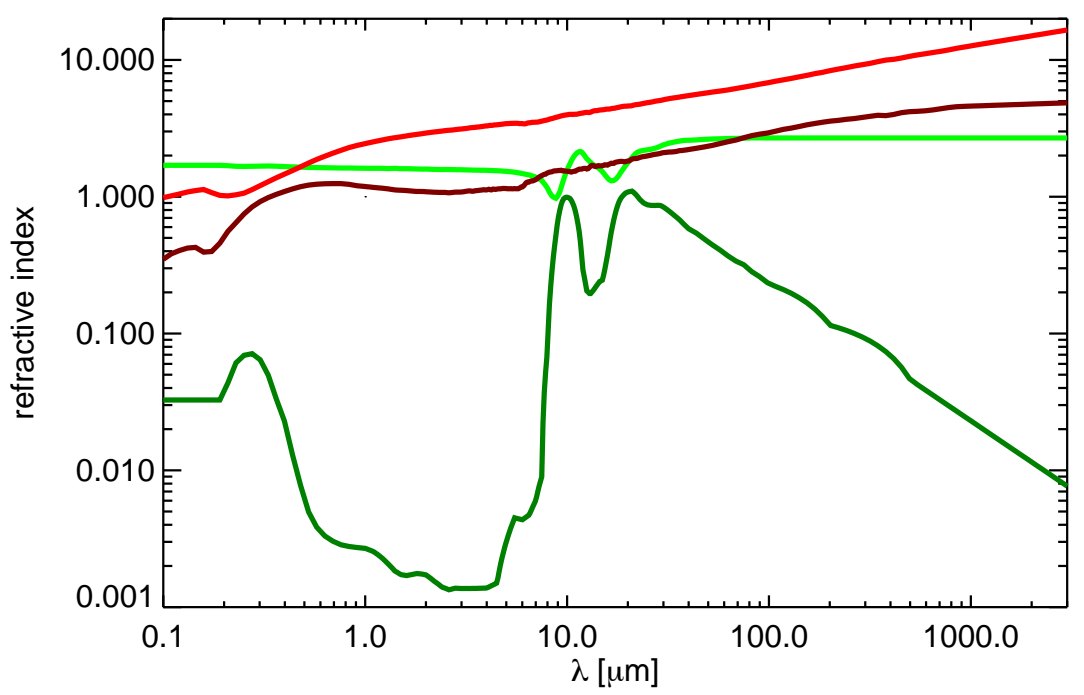

Figure 2. Laboratory measurements of the refractive index of silicate (green curves) and carbonaceous (red curves) materials (data from Dorschner et al. 1995; Zubko et al. 1996). The light red and green curves are the real part of the refractive index, the dark red and green curves the imaginary parts.

into their lattice. This makes for a less well-defined lattice, and the way in which the material was formed can now become important for the exact optical properties.

A very good place to start when looking for the optical properties of a large selection of cosmic dust materials is the database of measurements performed in Jena: http:/www.astro.uni-jena.de/ Laboratory/OCDB/.

As an example in Fig. 2 the refractive index of amorphous magnesium rich pyroxene and amorphous carbon are plotted as a function of wavelength.

For spectral resonances of crystalline materials, it is often useful to consider a theoretical oscillator model for the refractive index. Solid state features in crystalline materials are usually well described by the Lorentz oscillator model (see e.g. Bohren \& Huffman 1983). This model considers the refractive index due to harmonic lattice vibrations. The eigenfrequency of the harmonic oscillator is $\omega_{0}$. If there is only one eigenfrequency (resonance) this gives a refractive index

$$
m^{2}=m_{0}^{2}+\frac{\xi \omega_{p}^{2}}{\omega_{0}^{2}-\omega^{2}-i \gamma \omega} .
$$

In this equation $m_{0}$ is the real valued refractive index for $\omega \rightarrow \infty, \xi$ is the oscillator strength of the feature, $\omega_{p}$ is the plasma frequency, $\omega$ is the frequency of incident radiation and $\gamma$ is a damping factor. If $\omega$ is expressed in wavenumbers, we can express Eq. (26) in wavelengths by using $\omega=\lambda^{-1}$. If there are multiple eigenfrequencies, $m^{2}$ is a sum over different values of $\xi, \omega_{0}$ and $\gamma$. This model is used quite successfully, for example, for obtaining the refractive index from reflectance data.

Crystalline particles can have a different response when the electric field is applied along different crystallographic axes. So for these materials often refractive index data is provided for these different axes. To compute the optical properties of these materials, one actually has to take this into account. In practice this is often not computationally feasible, and one usually averages the computed optical 


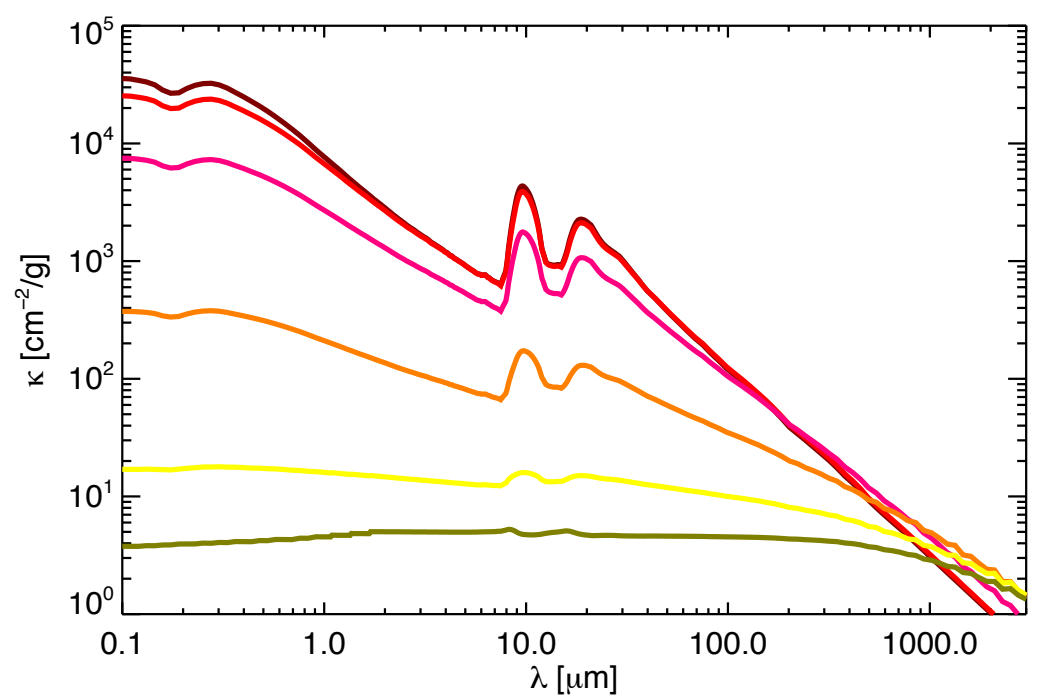

Figure 3. Mass absorption cross sections for a size distribution of particles with different slopes. The curves represent $p=5.0,4.5,4.0,3.5,3.0,2.5$ for the maroon, red, pink, orange, yellow, and olive curves respectively.

properties $\left(C_{\mathrm{abs}}, C_{\mathrm{sca}}, \mathbf{F}\right)$ over the three different crystallographic axes. DDA is one of the few methods that actually provides a natural way of dealing with material anisotropy.

\section{General dependence on particle properties}

The optical properties of a particle depend on the particle characteristics. Here we will discuss the general dependencies on particle size, material, and shape. Note that by shape we also refer to internal structure of the particle. The computations presented below are all based on the DIANA standards, as discussed in section 7. For understanding the dependencies the computational details are at this point not needed.

\subsection{Size dependence}

As we have seen before, for particles much smaller than the wavelength of radiation the absorption cross section scales with the volume of the particle (Eqs. 9-12), while the scattering cross section scales with the volume squared. At the other end of the size spectrum, i.e. particles that are much larger than the wavelength of radiation, we can simply use geometrical optics, meaning that the absorption and scattering cross sections both scale with the geometrical shadow of the particle. The extinction cross section for such large particles is wavelength independent and twice the geometrical shadow. Half of the extinction is made up of absorption and reflection, while the other half is caused by diffraction, i.e. distortion of the wavefront. The part in between these two extremes, i.e. where the size parameter $x$ is of order unity, is often called the resonance region. This is a very important diagnostic regime, since in both extreme cases the size of the particles is degenerate with the amount of material. As an example of the changes of the mass absorption cross section with increasing size, in Fig. 3 the opacity curves for different size distributions of particles are shown. For these curves 


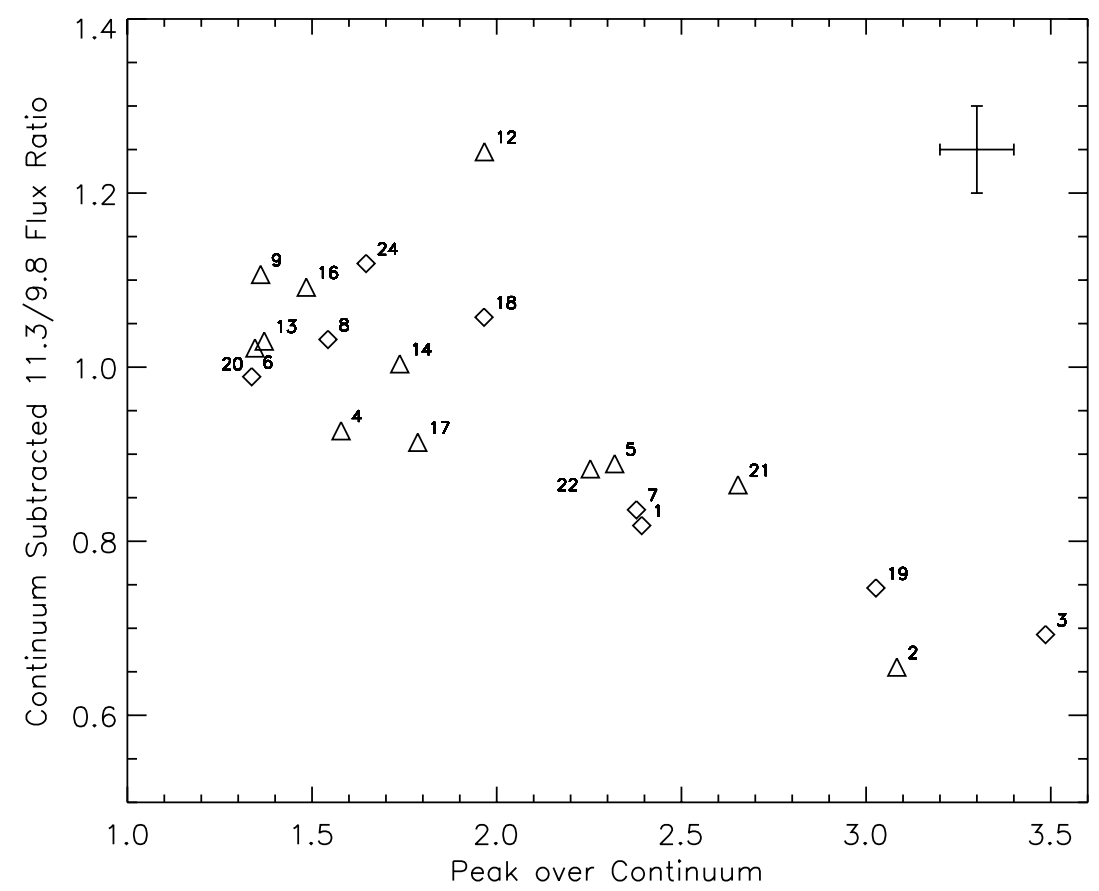

Figure 4. The strength of the $10 \mu \mathrm{m}$ silicate feature versus the shape for a selection of observed disks around Herbig stars. The strength on the $\mathrm{x}$-axis is measured as the peak over continuum, while the shape, represented by the continuum subtracted flux at 11.3 and $9.8 \mu \mathrm{m}$, is a measure for the broadness of the band. The figure is taken from (van Boekel et al. 2005, reproduced with permission (C) ESO), and the reader is referred to that paper for details on the exact definition of the quantities.

all particle properties are kept the same, but we skew the size distribution towards smaller or larger particle sizes. The effects this has will be discussed below.

The size distribution used in Fig. 3 is given by

$$
n(r) d r \propto \begin{cases}r^{-p}, & r_{\min }<r<r_{\max }, \\ 0, & \text { elsewhere }\end{cases}
$$

where we fix the minimum and maximum size of the distribution to $r_{\min }=0.05 \mu \mathrm{m}, r_{\max }=3000 \mu \mathrm{m}$. The curves correspond to different values of $p$, and thus to different weight of the small and large particle component (decreasing values of $p$ correspond to increasing weight of the large particles).

There are a few size indicators frequently used in the literature:

1. The solid state features

2. The anisotropy of scattering

3. The slope of millimeter emission

4. The degree of linear polarisation

We will discuss each of them here and indicate how these indicators change with increasing particle size. Below we will come back to these size indicators and also discuss how they are influenced by material properties and particle shape. 


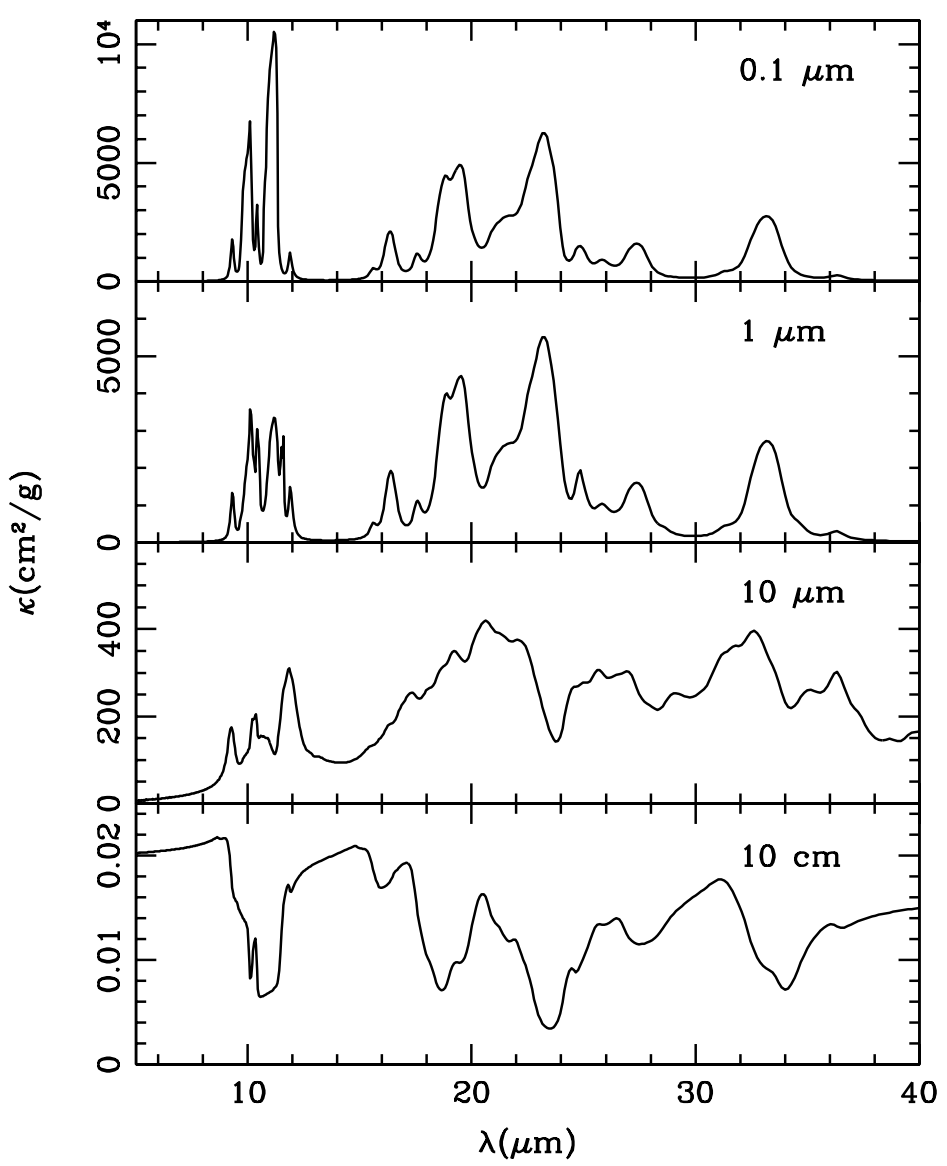

Figure 5. The mass absorption cross section for forsterite particles of different sizes. The computations are done using the DHS shape distribution. The weakening, and eventual inversion of the solid state resonances is clearly seen. Figure taken from Min (2009).

The solid state features: Since silicates are one of the dominant materials found in cosmic dust (at least in oxygen rich environments), the solid state features of silicate materials provide excellent tools for studying particle properties. The most common diagnostic used is the broad $10 \mu \mathrm{m}$ amorphous silicate feature observed in the emission spectra of almost all protoplanetary disks. To understand qualitatively what happens with a solid state feature, it is good to realize what the properties of a solid state feature are. In general it is a spectral location where the refractive index is increased (see also Fig. 2). Now, as we noted before, a particle can be said to be in the Rayleigh domain when the wavelength both inside and outside the particle is much larger than the particle itself, i.e. both $x \ll 1$ and $x|m| \ll 1$. These particle usually have a very high mass absorption (and thus emission) cross section. At the spectral location of a solid state resonance the refractive index is locally much higher, thus where for wavelengths away from the spectral resonance the particle is still in the Rayleigh limit, at the spectral location of the resonance the particle is not anymore $(x|m| \approx 1$, while $x<1)$. The net effect is that the absorption cross section at the wavelength of the spectral resonance decreases already 


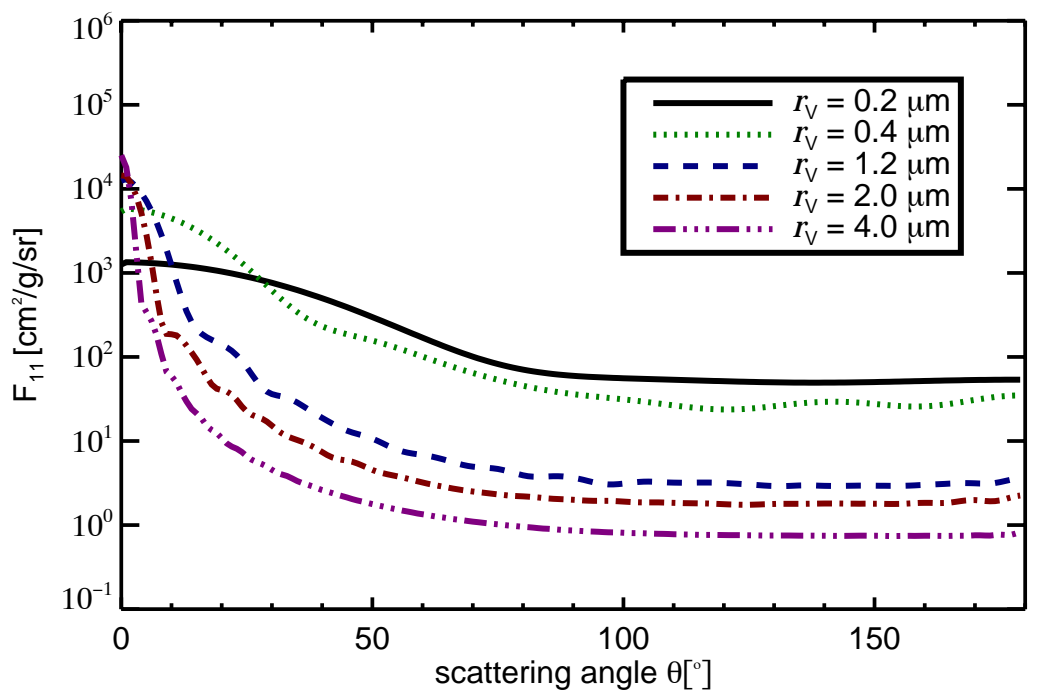

Figure 6. The phase function, $F_{11}$ for particles of different sizes. The computations are performed using DHS with $f_{\max }=0.8$.

at smaller values of $x$ then for wavelengths away from the resonance. Thus the net effect is that the spectral resonance decreases in strength.

With the above explanation it is easy to understand that very strong resonances, i.e. those with high values of $|m|$, will be affected by this for smaller particle sizes, as will spectral resonances at shorter wavelengths. This dependence of the spectral resonances on intrinsic feature strength and spectral location can be used to estimate particle sizes made up of crystalline material (see also Fig. 5 from Min et al. 2004). The complex dependence of the absorption cross section on size and refractive index also causes the $10 \mu \mathrm{m}$ amorphous silicate feature to broaden when the particle size increases. This broadening and weakening is an often used diagnostic to identify grain growth (for example see Fig. 4 from van Boekel et al. 2005).

The anisotropy of scattering: Very small particles scatter light in all directions almost isotropically. When the particle size increases, the scattering becomes increasingly forward peaked (see Fig. 6). Spatially resolved images of scattered light from a protoplanetary disk can therefore be used to determine particle sizes. The difference between the part of the disk tilted towards us and the part of the disk tilted away from us can be used to estimate the anisotropy of the scattering phase function (at least in the range of angles probed by the geometry of the system). This is commonly done for spatially resolved debris disks, which are optically and geometrically thin, making this analysis trivial. For protoplanetary disks the vertical extend of the disk (which is often unknown) and the effects of optical depth, make the analysis less straightforward. One has to be careful when relating the anisotropy of scattering observed in a limited range of scattering angles to a particle size. Where computational methods using smooth particles will give a generally good idea on the scattering anisotropy over the entire range of scattering angles, it can be different for realistic particles at intermediate to backward scattering angles. This will be discussed in more detail in the section on aggregates.

If we consider a macroscopic body, i.e. a particle for which we can apply geometrical optics, scattering consists of two basic terms: reflection and diffraction. Diffraction is a resonance process 


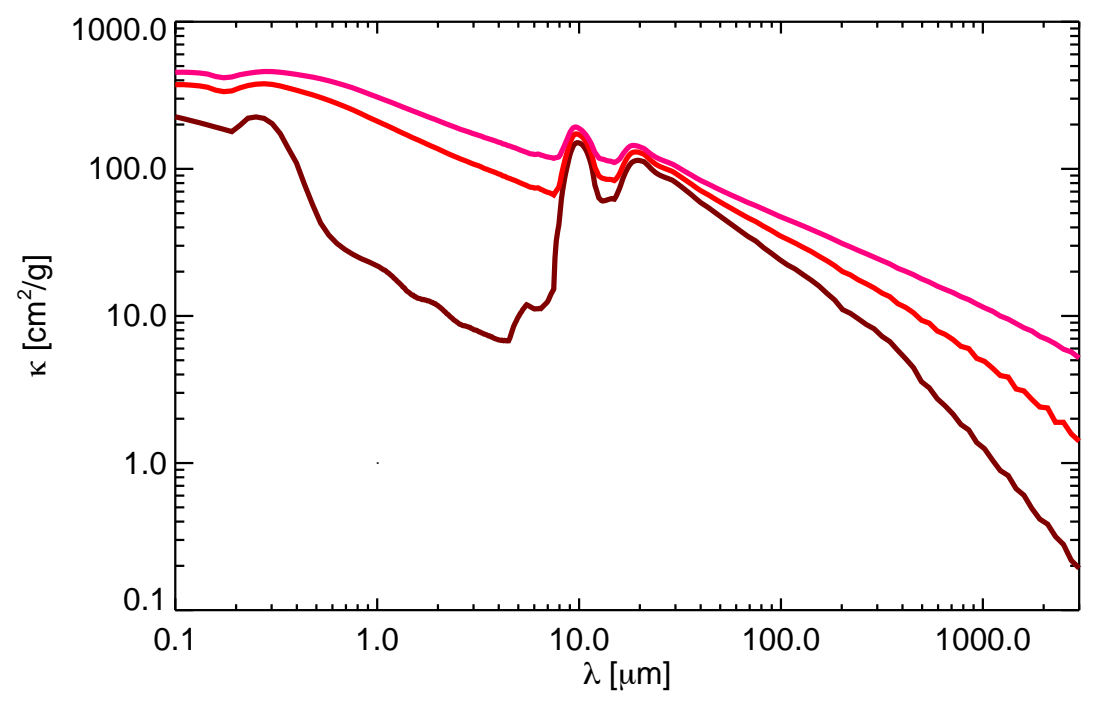

Figure 7. Mass absorption cross section as a function of wavelength for different mixtures of materials. The maroon, red, and pink curves correspond to 0,13 , and $25 \%$ amorphous carbon material by mass.

of disturbance of the incoming electromagnetic wave. It becomes increasingly forward peaked when increasing the geometrical shadow of the particle. For truly macroscopic objects it becomes so heavily forward peaked that it is not recognized as scattering anymore, since it basically does not alter the path of the incoming light beam, which is why for these bodies we only have to consider reflection.

The slope of millimeter emission: The absorption cross section of particle at millimeter wavelengths can usually be characterized relatively well by a single powerlaw,

$$
C_{\mathrm{abs}} \propto \lambda^{-\beta}
$$

For dielectric (i.e. non conducting) particles much smaller than the wavelength we have $\beta=2$. For conducting materials, like for example graphite, iron sulfide or metallic iron, the value of $\beta$ is usually much smaller. In the interstellar medium the value of $\beta \approx 1.7$ (Draine 2006). When the particle size increases, the value of $\beta$ decreases. For protoplanetary disks values of $\beta$ between 0.1 and 1.8 have been reported (see e.g. Lommen et al. 2010). These low values of $\beta$ are usually attributed to particle growth, but can also be partly explained by an increased amount of e.g. graphitic material. To see the change in slope when the grain size increases we plot in Fig. 3 a mixture of carbonaceous and silicate materials with a changing size distribution. The effect of adjusting the abundance of carbonaceous material is visualized in Fig. 7. Here it is very clear that an increasing amount of carbon can have a significant impact making the millimeter slope much shallower.

The degree of linear polarisation: Particles in the Rayleigh domain are very efficient polarizers. The provide a degree of linear polarization which is $100 \%$ at $90^{\circ}$ scattering. As the grain size increases, this degree of polarization is expected to drop. This is indeed the case for realistic particles. However, computations of smooth particles (like most methods provide) fail to reproduce this behavior. To understand why this is, we have to look at the polarizing effect of very large particles. For a very large particle we can use geometrical optics, i.e. Fresnel reflections. The Fresnel equations tell us that light reflected from a surface at the Brewster angle will be fully polarized. For a spherical particle 


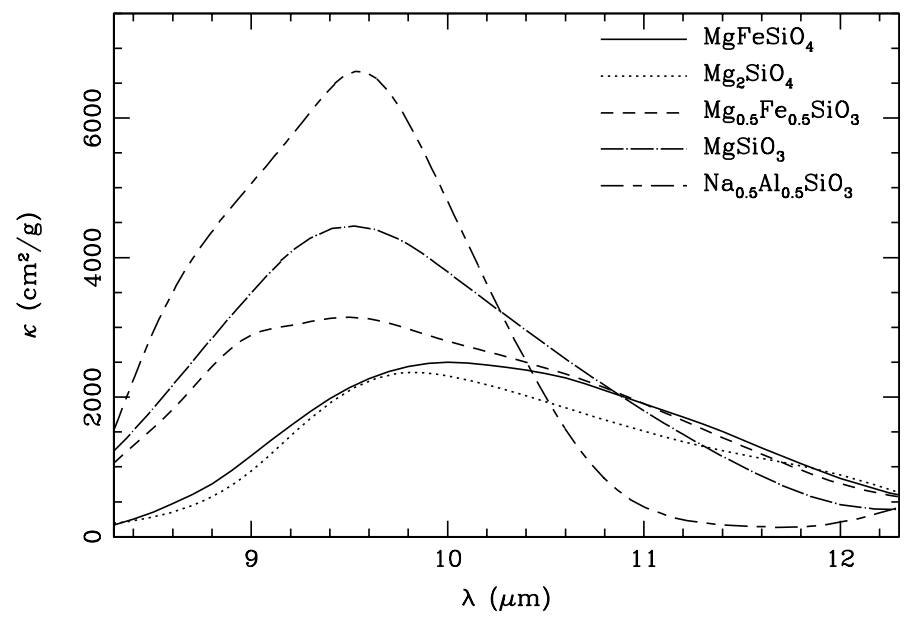

Figure 8. The $10 \mu \mathrm{m}$ amorphous silicate feature for different compositions of the silicate. Figure taken from (Min et al. 2007, reproduced with permission (C) ESO).

this corresponds to a scattering angle of $\theta_{\text {Brew }}=\pi-2 \arctan (m)$. For silicate material in the optical part of the spectrum $m \approx 1.6$, i.e. $\theta_{\mathrm{Brew}} \approx 64^{\circ}$. At this angle a smooth surface spherical particle will display $100 \%$ polarization. For other smooth surface convex particles this angle can be slightly different, but will always exist. However, when the surface is not smooth, the polarization will be imperfect and much lower, like what is detected for realistic particles in the laboratory, but also from rough surface scattering (i.e. the polarization phase curve of atmosphereless solar system bodies). The conclusion is that there is certainly potential in using the degree of polarization, but that using it requires very careful modeling of particle shape and surface structure. We will return to the topic of polarization when discussing aggregate particles.

\subsection{Material dependence}

Different materials display different spectral structure in the opacities. Details in the location and width of solid state features can be attributed to differences in material properties. For example the $10 \mu \mathrm{m}$ amorphous silicate feature is very dependent on the exact composition of the silicate (see Fig. 8). Also, the more general behavior in the optical part of the spectrum can be very different. Amorphous silicates with only magnesium in the lattice have a very low absorption efficiency, while the inclusion of a small amount of iron atoms can boost the absorption significantly. This is very important for the heating of cosmic dust particles.

In protoplanetary disks the most important absorber in the optical is expected to be carbonaceous material of any kind (amorphous or graphitic). In Fig. 7 we display the effect of including an increasing amount of amorphous carbon into the dust mixture. Besides the already discussed change of the millimeter opacity slope, there is also a significant increase of the absorption efficiency at visible wavelengths. 


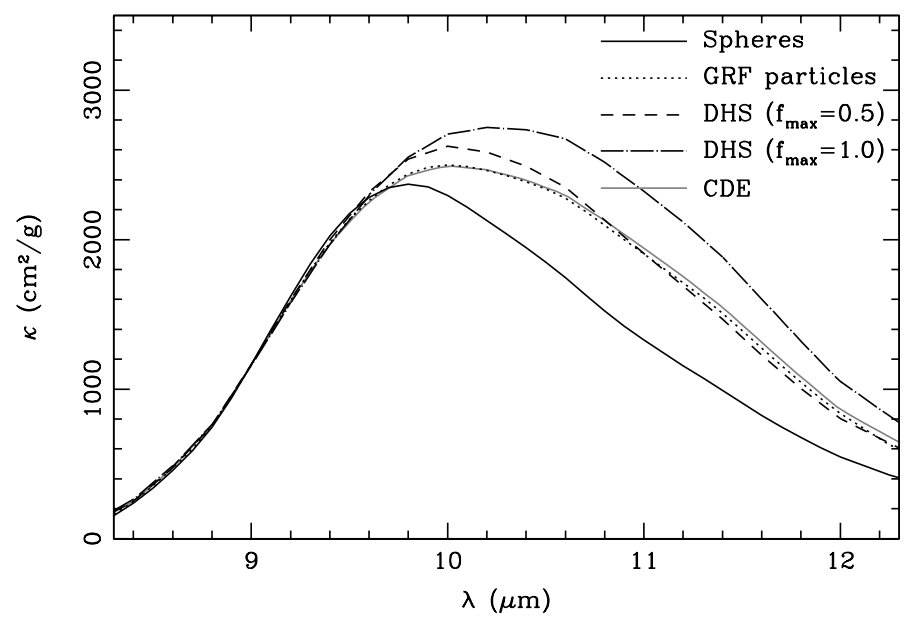

Figure 9. The shape of the $10 \mu \mathrm{m}$ silicate feature for different particle shape distributions. We plot the feature for homogeneous spheres, Gaussian Random Field (GRF) particles (see Min et al. 2007, for details), DHS particles with different values of $f_{\max }$, and CDE. The shift and broadening of the feature for increasing deviations of perfect spherical particles is very clear. Figure taken from (Min et al. 2007, reproduced with permission (C ESO).

\subsection{Shape dependence}

Particles of different shapes produce different opacities. The simplest shape one can consider is that of a homogeneous sphere, for which the full solution of Maxwell's equations is easily written down. However, a homogeneous sphere has been shown to be a poor representation of cosmic dust particles in general (e.g. Fabian et al. 2001). The main reason for this is that a perfect sphere exhibits resonances that do not occur in nature. A commonly heard remark is that a size distribution will wash out these resonances. However, although a size distribution might mask the resonances by smoothing them, they do not go away.

The most important effects of particle shape are seen when the refractive index is relatively high. In these cases particle shape becomes a crucial parameter. This manifests itself in two important aspects: the solid state features, and the millimeter opacity of conducting materials.

Solid state features are locations in the spectrum where the refractive index locally increases. As can be seen from Eq. 11 the maximum absorption efficiency of an ellipsoid depends on the value of $L$. Since the refractive index at the spectral location of a feature rapidly changes, it goes through all different values, and thus crosses the different maxima for different values of $L$. This means that the exact location of the solid state resonance is a strong function of $L$ (or the shape of the form-factor distribution). This effect is strongest for the very strong resonances found for crystalline materials, but it is also very clear for amorphous silicates (see Fig. 9).

Conducting (or semi-conducting) materials can be considered as materials with a spectral resonance at $\omega_{0}=0$ (or very small values of $\omega_{0}$; see Eq. 26). Thus at long wavelengths the refractive index increases towards relatively high values. This implies that, similar to the shape dependence of the spectral resonances, the millimeter slope of these materials becomes highly dependent on particle shape. This is illustrated in Fig. 10 where we plot the absorption efficiencies for a standard mixture of silicate and carbon, and for pure carbon grains for different values of $f_{\max }$. Due to the use of effective medium theory, the refractive index of the material mix at long wavelengths is not very high 
Summer School "Protoplanetary Disks: Theory and Modeling Meet Observations"
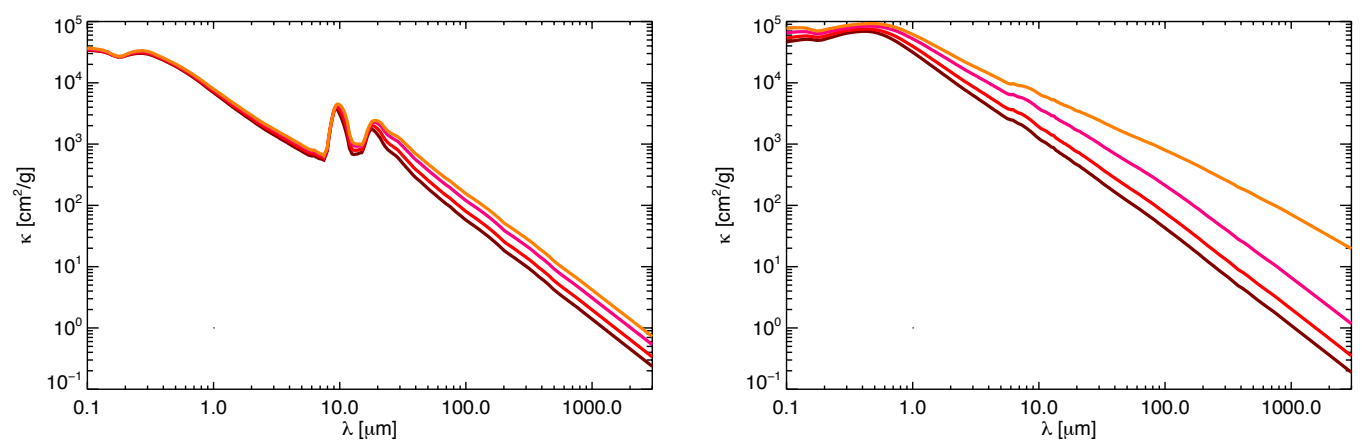

Figure 10. Mass absorption cross sections as a function of wavelength for different values of the irregularity parameter in the DHS shape distribution. Left figure shows a standard silicate carbon mixture, while right figure is for pure carbon grains. Maroon, red, pink, and orange curves correspond to $f_{\max }=0,0.4,0.8,1.0$ respectively.

(the carbon abundance is relatively low), thus the millimeter opacity slope is not heavily influenced. However, for the pure carbon grains, the slope in the millimeter changes significantly when $f_{\max }$ is increased. Amorphous carbon is still a semi-conducting material, i.e. its refractive index does not reach extreme values. If we would consider more extreme materials, like metallic iron, these effects are even stronger.

The difficulty with this strong dependence on particle shape is that it is impossible to exactly model the particle shapes that we expect. The small scale substructure is essential, so only considering the external shape of the particle (which is usually rather simpel, or even spherical) is not enough. However, how strong the effects of substructure really are is a question that has no simple answer. The best solution for this is to consider laboratory measurements of powder materials and fit these using a shape distribution. This has been done by Mutschke et al. (2009), who fitted a form-factor distribution to measurements of powder spectra. These powder spectra are also well represented by a DHS shape distribution with $f_{\max } \approx 0.8$. For very high refractive indices the maximum absorption cross section is obtained for extremely low values of the form-factor $L$. Thus, for computations of these materials, the detailed shape of the form-factor distribution at these very low $L$-values is crucial. However, these extremely high refractive indices are usually not probed by solid state spectral resonances. Thus, a model that reproduces the solid state spectral resonances very well is not automatically successful at representing the optical properties of (semi)conducting materials at millimeter wavelengths (see also Fig. 2).

\section{General properties of aggregates}

Dust grains that enter the protoplanetary disk from the molecular cloud will stick together and form larger structures in the high density regions of protoplanetary disks. The optical properties of such dust aggregates can be significantly different from those of single, compact grains. This can be best understood when one looks at the fundamental property of an aggregate; it basically consists of two different scales, one for the monomers and one for the aggregate as a whole. These two different scales will show up in the optical properties of aggregates and determine their apparent size under different observing conditions. Below we will discuss how the different size indicators connect to these two different scales in an aggregate particle. These insights are based on studies by Kataoka et al. (2014); 
Kimura et al. (2006); Min et al. (2006a); Volten et al. (2007) and DDA computations of aggregate particles from Min et al. in prep.

Kataoka et al. (2014) discuss the opacity evolution of very fluffy aggregates with particle size and filling factor using effective medium theory. They conclude that for very fluffy aggregates the absorption (and thus emission) properties of aggregates are determined by the factor $f \cdot r$, with $f$ the filling factor and $r$ the outer radius of the aggregate. Aggregates with the same $f \cdot r$ values have the same mass absorption cross section. For scattering, this is somewhat different and the interested reader is referred to their paper for details. They also provide an analytical formula for the opacities of aggregate particles, which should be used with care, as one of the main assumptions going in is the assumption that the monomers are perfect spheres, which influences among others the millimeter slope when conducting materials are present.

\subsection{The solid state features}

The $10 \mu \mathrm{m}$ silicate feature of aggregates of different fractal dimension was studied in detail by Min et al. (2006a). The conclusion of this study is that for the same aggregate mass (so the same $r_{V}$ ) the feature is stronger when the fluffyness of the aggregate is increased (i.e. its fractal dimension is decreased). Fig. 11 (taken from Min et al. 2006a) displays this very clearly. The way to understand this is that the particle needs to become 'optically thick' for the feature to decrease in strength. Since the average density of a very fluffy aggregate is much lower than for a compact aggregate with the same mass, its optical depth is also smaller. Note here that we can use the concept of optical depth only as a way to understand this effect. The interaction of light with a particle should strictly be described in terms of the Maxwell equations, and only for very large particles this can be done using radiative transfer concepts.

\subsection{The anisotropy of scattering and degree of linear polarisation}

As discussed above, for very large particles scattering is made up of a diffraction term and a reflection term. The scattering phase function of aggregate particles can be understood from these two terms qualitatively. Since the diffraction term only depends on the size of the geometrical shadow, it is not dependent on the exact shape of the particle but only on the external size of the aggregate as a whole. This term causes an increasing forward scattering with increasing aggregate size. At intermediate to backscattering angles the scattering of a large particle is dominated by reflection. However, for an aggregate particle the substructure caused by the monomers of the aggregate is crucial, so we cannot consider this part of the scattering as reflection off a smooth surface. This part of the scattering term is caused by the monomers of the particle and the 'surface' they create. Computations and laboratory measurements show that this part of the scattering is indeed dominated by the properties of the monomers of the aggregate (e.g. Volten et al. 2007). This implies that also the degree of linear polarization at intermediate scattering angles is completely dominated by what the monomers do. Compare this to the polarization behavior of very large smooth particles, displaying $100 \%$ polarization around the Brewster angle, and it is clear that this can be very different (see also Fig. 12 taken from Min et al. in prep).

\section{DIANA standards}

Modeling a protoplanetary disk requires many parameters. To make a meaningful comparison between different modeling efforts it is good to realize that changing the assumptions going into the 


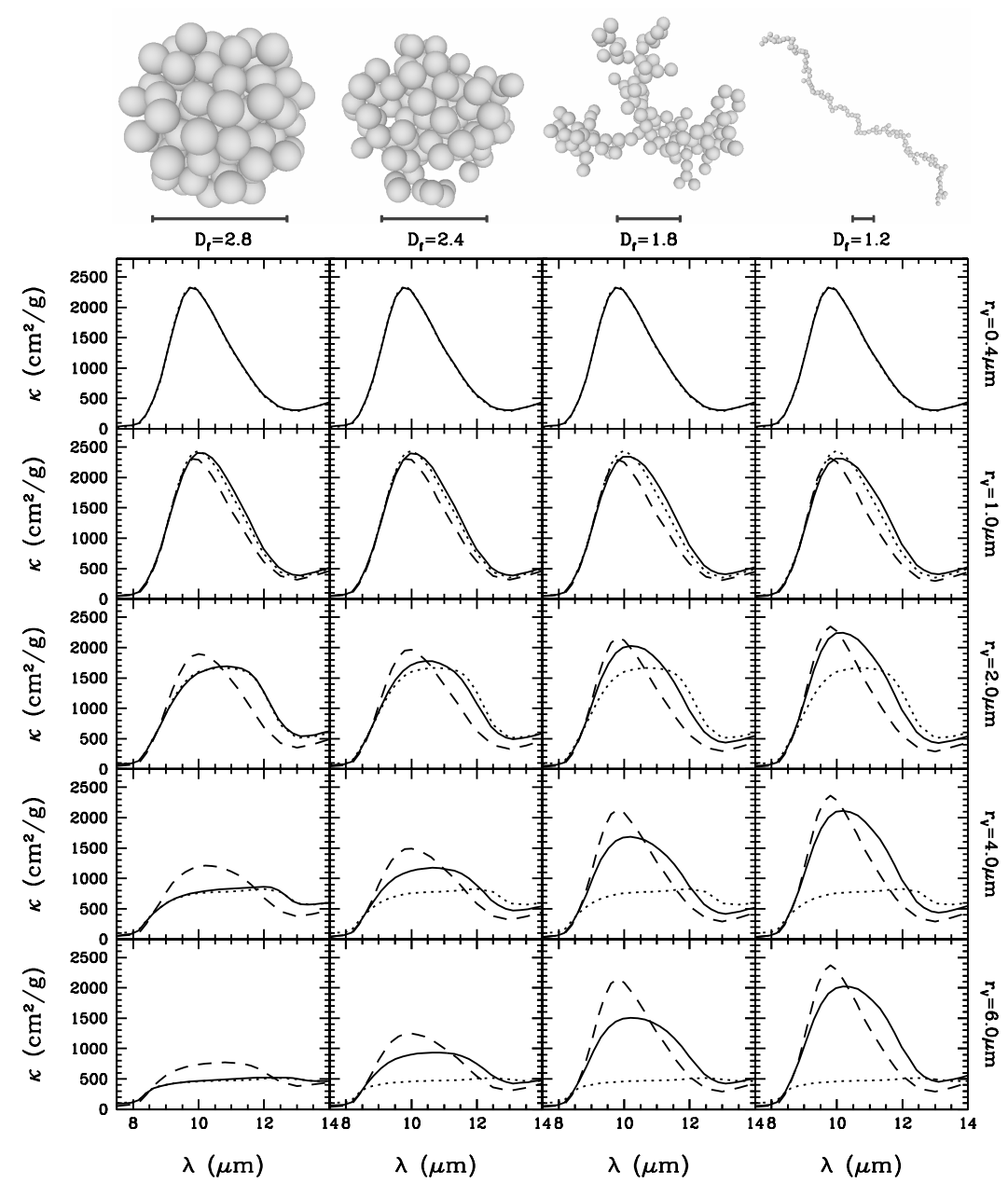

Figure 11. The $10 \mu \mathrm{m}$ absorption spectra of aggregate particles for different volume equivalent radii. The solid curves show the computations for the aggregate particles, the dotted curves for homogeneous spheres with the same size, and the dashed curves display a porous sphere approximation. The upper panels show graphical representations of the aggregates with $r_{V}=2 \mu \mathrm{m}$, the bar below the aggregates displays a measure for the scale of the image by showing the diameter of a volume equivalent sphere. Figure taken from (Min et al. 2006a, reproduced with permission (C) ESO).

modeling significantly affects the outcome. The optical properties of the dust grains stand at the basis of any modeling attempt; they completely dominate the spectral appearance and temperature structure. It is therefore a very difficult task to compare modeling efforts conducted with completely different assumptions on the properties of the dust grains. Within the DIANA project we recognize this issue, and therefore propose to use a modeling standard for the opacities of the dust grains that is easily accessible for modelers, requires little knowledge on the details, and is computationally not too expensive. This modeling framework is outlined below. 

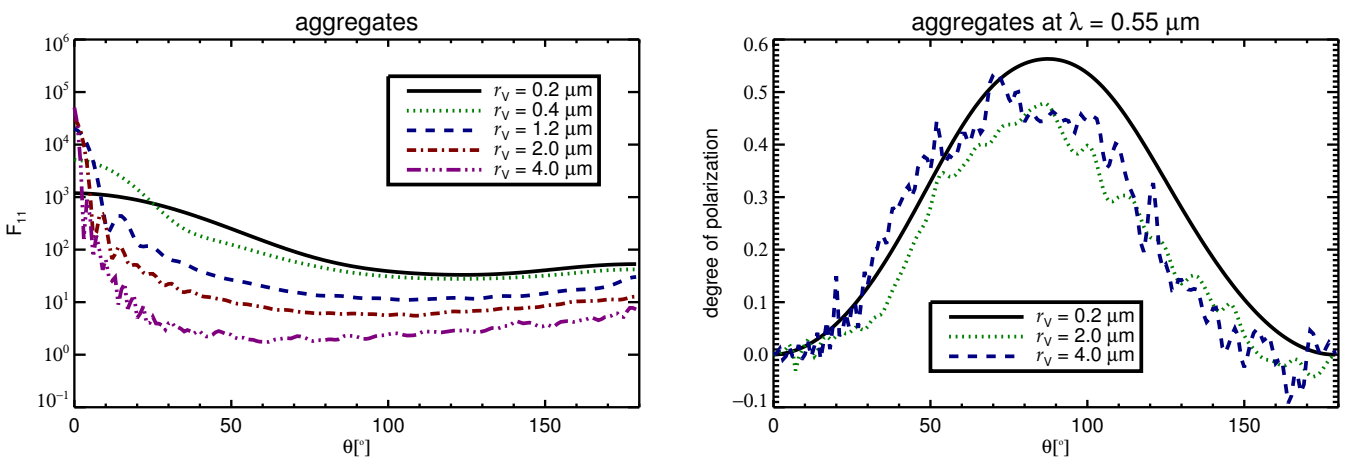

Figure 12. Phase function, $F_{11}$ (left), and polarization curve, $-F_{12} / F_{11}$ (right), computed for aggregate particles of different sizes.

\subsection{Dust size distribution}

For the dust size distribution we take a simple powerlaw with minimum size $r_{\min }=0.05 \mu \mathrm{m}$ and maximum size $r_{\max }=3000 \mu \mathrm{m}$ according to Eq. 27 with the power of the powerlaw, $p$, a free parameter.

\subsection{Dust shape distribution}

We choose to adopt the Distribution of Hollow Spheres, DHS Min et al. (2005). This has the advantage that computations can be carried out quite efficiently and the accuracy of the computations is greatly enhanced with respect to perfect spheres. The DHS has one shape parameter, which is the maximum volume fraction of the inner vacuum cavity, $f_{\max }$.

\subsection{Composition}

The composition of the grains is taken to be a mixture of amorphous silicates and amorphous carbon. All other components are not included in the standard setup. Even though iron sulfide is a very likely component of protoplanetary dust, for simplicity we assume the optical properties of all featureless components are well represented by a single continuum opacity source which we take to be amorphous carbon. For the interpretation of the results, this has to be kept in mind, the amorphous carbon represents all unknown, featureless opacity sources.

In addition to these two materials, we mix in a fraction of vacuum, to simulate porosity. We take $25 \%$ of the volume to be vacuum, which is a rather mild porosity. Among others, this influences the strength of the 10 micron silicate feature.

We mix everything together using the Bruggeman mixing rule (Eq. 14 with $m_{m}=m_{\text {eff }}$ ), which can be written as,

$$
\sum_{i=1}^{N} w_{i} \frac{m_{i}^{2}-m_{\mathrm{eff}}^{2}}{m_{i}^{2}+2 m_{\mathrm{eff}}^{2}}=0,
$$

To mix in vacuum, one has to simply mix in a material with $m_{i}=1$. The Bruggeman effective medium theory has no closed form solution, so a solution has to be found by iterative, numerical methods.

The silicate composition is likely somewhere in between pyroxene and olivine, with a rather high $\mathrm{Mg} / \mathrm{Fe}$ ratio. This has been found for the interstellar silicates by looking at the interstellar silicate 
feature. Also, this is likely from dust condensation. Finally, ironsulfide is probably a drain of Fe, which also favors $\mathrm{Mg}$ rich silicates. We follow the composition of interstellar silicates as derived by Min et al. (2007), which has $\mathrm{Mg} /(\mathrm{Mg}+\mathrm{Fe}) \approx 0.7$. For simplicity we only consider the pyroxene stoichiometry.

The refractive index data we take from

- Silicate: $\mathrm{Mg}_{0.7} \mathrm{Fe}_{0.3} \mathrm{SiO}_{3}$ from Dorschner et al. (1995) (material density $\rho=3.01 \mathrm{~g} / \mathrm{cm}^{3}$ )

- Carbon: from Zubko et al. (1996), sample BE (we take the material density $\rho=1.80 \mathrm{~g} / \mathrm{cm}^{3}$ )

There are several studies reporting on refractive index data for carbonaceous material. The refractive indices are often different, which can be attributed to different lattice structures of the carbon. The choice of the carbon material we propose comes from the following considerations. First, the data has to be smooth, featureless. Second, conducting materials like carbon, graphite, iron sulfide, or metallic iron, have increasing refractive index at long wavelengths. This heavily influences the $\mathrm{mm}$ slope of the opacities. However, this effect is not visible in all measurements of amorphous carbon, but we want to select one that does. These considerations are very practical, i.e. the data has to be able to reproduce our observations.

Towards wavelengths shorter than available from the measurements, one could in principle extrapolate using the equations given by Bohren \& Huffman (1983, page 234)

$$
n=1-\frac{\omega_{p}^{2}}{2 \omega^{2}}, \quad k=\frac{\gamma \omega_{p}^{2}}{2 \omega^{3}},
$$

with $n$ and $k$ the real and imaginary part of the refractive index. The parameters $\omega_{p}$ and $\gamma$ are obtained from the first datapoint available. However, we find that for the materials we use, this does not give a logical extrapolation. Therefore, we take the refractive index to be constant for the small range we have to extrapolate towards smaller wavelengths.

For wavelengths longer than available from the measurements, we use a loglog extrapolation. We decide not to use the general extrapolation formula for dielectrics since this is inaccurate for semiconducting materials like carbon.

The carbon fraction determines in part the strength of the silicate feature and greatly influences the opacity slope at millimeter wavelengths. We take this to be a free parameter in the modeling. Useful values are between 10 and $20 \%$ by mass. An important characteristic influenced by the amount of carbon is the slope of the mm opacity, $\beta$. This varies from $\beta=1.8$ for $5 \%$ carbon to $\beta=1.4$ when using $20 \%$ carbon for the smallest grain sizes. The value of $\beta$ is also influenced by grain size (decreasing with increasing grain size).

\subsection{Summary of the standards}

When modeling protoplanetary disks, there are parameters of the dust grains that we want to vary, and parameters that we want to fix. We fix the shape distribution and materials we use, while the size distribution and carbon content are parameters that we can vary.

For the particle shape we use the DHS model. We include porosity and mix the materials into one effective material using the Bruggeman mixing rule. All fixed and free parameters are summarized in Table 1.

\section{Summary}

The optical properties of particles in protoplanetary disks are of crucial importance for studying and modeling them. They are heavily dependent on particle size, shape, and composition. We described 
Table 1. The parameters used for the standardized optical properties used in the DIANA modeling framework. The parameter ranges given for the free parameters are only indications of reasonable values.

\begin{tabular}{|c|c|}
\hline \multicolumn{2}{|l|}{ fixed parameters } \\
\hline minimum size $\left(r_{\min }\right)$ & $0.05 \mu \mathrm{m}$ \\
\hline maximum size $\left(r_{\max }\right)$ & $3000 \mu \mathrm{m}$ \\
\hline irregularity parameter $\left(f_{\max }\right)$ & 0.8 \\
\hline vacuum fraction $\left(w_{\mathrm{vac}}\right)$ & 0.25 (by volume) \\
\hline \multicolumn{2}{|l|}{ optical data used } \\
\hline Silicate $\left(\rho=3.01 \mathrm{~g} / \mathrm{cm}^{3}\right), \mathrm{Mg}_{0.7} \mathrm{Fe}_{0.3} \mathrm{SiO}_{3}$ & Dorschner et al. (1995) \\
\hline Carbon $\left(\rho=1.80 \mathrm{~g} / \mathrm{cm}^{3}\right)$, sample BE & Zubko et al. (1996) \\
\hline \multicolumn{2}{|l|}{ free parameters } \\
\hline powerlaw $(p)$ & $\approx 2.5$ to 4.5 \\
\hline carbon fraction $\left(w_{\text {carbon }}\right)$ & $\approx 10$ to $20 \%$ by mass \\
\hline
\end{tabular}

above how these dependencies work in general, and where one has to be extra careful in drawing conclusions. We describe an approach into computing optical properties for protoplanetary dust that takes into account most of these considerations, and provides a starting framework for studies of dust in protoplanetary disks.

The wealth of complications arising when computing optical properties have in the past discouraged people from using anything different than the most simple approach. We hope to have provided enough insight into this complex problem to provide handles into deviating just enough from this oversimplified approach and to use appropriate approximations and insights to arrive at more robust and balanced conclusions.

Acknowledgements The research leading to these results has received funding from the European Union Seventh Framework Programme FP7-2011 under grant agreement no 284405.

\section{References}

Bohren, C. F. \& Huffman, D. R. 1983, Absorption and scattering of light by small particles

Dorschner, J., Begemann, B., Henning, T., Jaeger, C., \& Mutschke, H. 1995, A\&A, 300, 503

Draine, B. T. 1988, ApJ, 333, 848

Draine, B. T. 2006, ApJ, 636, 1114

Dubovik, O., Sinyuk, A., Lapyonok, T., et al. 2006, Journal of Geophysical Research (Atmospheres), 111,11208

Fabian, D., Henning, T., Jäger, C., et al. 2001, A\&A, 378, 228

Kataoka, A., Okuzumi, S., Tanaka, H., \& Nomura, H. 2014, A\&A, 568, A42

Kimura, H., Kolokolova, L., \& Mann, I. 2006, A\&A, 449, 1243

Li, A. 2008, ArXiv e-prints 
Summer School "Protoplanetary Disks: Theory and Modeling Meet Observations"

Lommen, D. J. P., van Dishoeck, E. F., Wright, C. M., et al. 2010, A\&A, 515, A77

Mie, G. 1908, Annalen der Physik, 330, 377

Min, M. 2009, in Astronomical Society of the Pacific Conference Series, Vol. 414, Cosmic Dust Near and Far, ed. T. Henning, E. Grün, \& J. Steinacker, 356

Min, M., Dominik, C., Hovenier, J. W., de Koter, A., \& Waters, L. B. F. M. 2006a, A\&A, 445, 1005

Min, M., Dominik, C., \& Waters, L. B. F. M. 2004, A\&A, 413, L35

Min, M., Hovenier, J. W., \& de Koter, A. 2003, A\&A, 404, 35

Min, M., Hovenier, J. W., \& de Koter, A. 2005, A\&A, 432, 909

Min, M., Hovenier, J. W., Dominik, C., de Koter, A., \& Yurkin, M. A. 2006b, J. Quant. Spec. Radiat. Transf., 97, 161

Min, M., Hovenier, J. W., Waters, L. B. F. M., \& de Koter, A. 2008, A\&A, 489, 135

Min, M., Waters, L. B. F. M., de Koter, A., et al. 2007, A\&A, 462, 667

Mishchenko, M. I., Travis, L. D., Kahn, R. A., \& West, R. A. 1997, J. Geophys. Res., 102, 16831

Mutschke, H., Min, M., \& Tamanai, A. 2009, A\&A, 504, 875

van Boekel, R., Min, M., Waters, L. B. F. M., et al. 2005, A\&A, 437, 189

van de Hulst, H. C. 1957, Light Scattering by Small Particles

Volten, H., Muñoz, O., Hovenier, J. W., et al. 2007, A\&A, 470, 377

Voshchinnikov, N. V., Videen, G., \& Henning, T. 2007, Appl. Opt., 46, 4065

Zubko, V. G., Mennella, V., Colangeli, L., \& Bussoletti, E. 1996, MNRAS, 282, 1321 
